\title{
Study on the Fault Diagnosis for Scroll Compressor Based on Information Entropy
}

\author{
Liu Tao \\ College of Mechanical and Electrical \\ Engineering \\ Lan Zhou University of Technology \\ Lan Zhou, China \\ cathyliu1999@126.com
}

\author{
Wu Zai xin \\ College of Mechanical and Electrical \\ Engineering \\ Lan Zhou University of Technology \\ Lan Zhou, China \\ wu_zaixin@sina.com
}

\author{
Wang Yong wei \\ College of Mechanical and Electrical \\ Engineering \\ Lan Zhou University of Technology \\ Lan Zhou, China, 18215191551 \\ beijingyongwei@126.com
}

\begin{abstract}
In order to make a quantitative description for the running status of the scroll compressor, a fault diagnosis method combined with the theory of entropy and grey incidence degrees in information theory is set up. Based on singular spectrum entropy in time domain, power spectrum entropy in frequency domain, wavelet power spectrum entropy and wavelet space feature spectrum entropy in time-frequency domain, the method is used as the comprehensive indicator to evaluate of the vibration status of scroll compressors. An example proves that the method is effective and can be used to identify several kinds of fault state of scroll compressor successfully.
\end{abstract}

Keywords-scroll compressor; information entropy; grey incidence degrees; fault diagnosis

\section{INTRODUCTION}

Scroll compressors have attracted great attention in recent years. They have been widely used in air conditioning, refrigeration, a variety of gas compression engine supercharger and booster pumps and other systems due to the advantages of more compact structure, higher efficiency, lower noise level and higher reliability. However, Failure analysis of working process of scroll compressors is still in the primary stage in which the working state was described with traditional single spectrum analysis. The performance data of operating conditions can't be calculated through accurate mathematical models.

In recent years, there is a very broad application in the test system platform build and noise and vibration analysis for scroll compressor. But this is still difficult to meet the requirements of the scroll compressor condition monitoring. Although Peng Bin [2] has proposed test systems based on modular hardware approach to obtain the operation of the scroll compressor, the exceptions of the operating conditions was not analyzed. Zhen-quan Liu [3] has analyzed the vibration signal of the scroll compressor in time domain and frequency domain, but the signal is vulnerable to the influence of the field interference. Shao Hua [4] found the source of vibration of the scroll compressor through the LabVIEW development platform. However, the reliability of fault diagnosis is weak because the correspondence between the frequency domain analysis of vibration signal and fault symptoms are often affected by sampling time, testing position, and sampling frequency.

Sponsors : National Natural Science Foundation of China (Grant No. 50965011) and National Science Foundation of Gansu Province in China (Grant No. 1014RJZA025)
Based on extracting the time domain, frequency domain, time-frequency domain fault feature, the fault discrimination method of scroll compressors based on gray relational degree is described in the paper. Furthermore, it is used as a comprehensive indicator to evaluate the scroll compressor vibration state.

\section{INFORMATION ENTROPY CHARACTERISTICS OF THE VIBRATION SIGNAL}

\section{A. Singular spectrum entropy}

Singular spectrum entropy method is a good tool to analyze sequences which has less sampling points [5] and containing noise. For a given time series $\left[\begin{array}{llll}x_{1} & x_{2} & \cdots & x_{N}\end{array}\right]$ and window length $\mathrm{M}$, the trajectory matrix of the embedding space $m$ rows $M$ column can be constructed as

$$
A=\left[\begin{array}{cccc}
x_{1} & x_{2} & \cdots & x_{M} \\
x_{1+\tau} & x_{2+\tau} & \cdots & x_{M+\tau} \\
\vdots & \vdots & & \vdots \\
x_{1+(N-M) \tau} & x_{2+(N-M) \tau} & \cdots & x_{N}
\end{array}\right],
$$

where $m=N-M+1, N=M+(m-1) \times \tau$

After the trajectory matrix is transformed, its lagged covariance is a $M \times M$ matrix $S$. $S$ is defined as

$$
S=\left[\begin{array}{cccc}
s(0) & s(1) & \cdots & s(M-1) \\
s(1) & s(0) & \cdots & s(M-2) \\
\cdots & \cdots & \cdots & \cdots \\
s(M-1) & s(M-2) & \cdots & S(0)
\end{array}\right] .
$$

The singular value decomposition is carried on the matrix S. Assume that the singular values satisfy $\delta_{1} \geq \delta_{2} \geq \cdots \geq \delta_{M}$, $\left\{\delta_{i}\right\}$ constitutes the singular value spectrum of the vibration signal. So it is reckoned that the singular value spectrum of $\left\{\delta_{i}\right\}$ is a division of the vibration signal in time domain [6]. 
Singular spectrum entropy in the time domain signal can be defined by

$$
\begin{aligned}
& S_{1}=-\sum_{i=1}^{M} p_{i} \log p_{i}, \\
& p_{i}=\delta_{i} / \sum_{i=1}^{M} \delta_{i} .
\end{aligned}
$$

Where $p_{i}$ is the proportion of the $\mathrm{i}$-th singular value in the entire singular value spectrum. Singular spectrum entropy reflects the uncertainty of the unit vibration energy. The simpler the signal, the more the energy is concentrated on a few modes. On the contrary, the more complex the signal, the more the energy is dispersed.

\section{B. Power Spectral Entropy}

A time window $w(n)$ is defined to intercept signal $x(n)$. The intercepted signal segment is $x_{n} \cdot w(n)$. Calculate the power spectrum value of the signal within the window and observe the variation of power spectral entropy according to the shift of the window. Assume short-time window of length is $M$, the sliding step of length is $\sigma$, and data sequence is divided into $K$ segments, the average energy within the window is given by

$$
\begin{aligned}
& U_{i}=\frac{1}{M}\left|\sum_{n=0}^{M-1} x^{(i)}(n) w(n) e^{-j \omega n}\right|^{2} \quad i=1,2, \cdots, K, \\
& (K=(N-M) / \sigma)
\end{aligned}
$$

The power spectral entropy of signal in frequency domain can be defined as

$$
\begin{aligned}
& S_{2}=-\sum_{i=1}^{K} S^{\prime}, \\
& S^{\prime}=q_{i} \log _{2} q_{i} \text { and } q_{i}=U_{i} / \sum_{i=1}^{K} U_{i} .
\end{aligned}
$$

Where $q_{i}$ is the proportion of the i-th energy window spectrum value in the entire power spectrum. The more uniform the vibrational energy distribution in the entire frequency, the more complex the signal is, and the greater the degree of uncertainty is.

\section{Spatial characteristics of wavelet spectral entropy and Wavelet energy spectrum entropy}

Assume that the signal is decomposed into $D_{j}$ with the wavelet decomposition at scale $j(j=1,2 \cdots M)$. The decomposition results can constitute a $M \times N$ matrix $D_{M \times N}$, if the decomposition number is $m$. According to the signal singular value decomposition theory, the singular value decomposition is done on wavelet transform coefficient matrix $D_{M \times N}$ to get singular value spectrum $\left\{\delta_{i}^{\prime}\right\}$. The time- frequency domain wavelet space characteristic spectral entropy is defined by

$$
S_{3}=-\sum_{j=1}^{M} p_{j}^{\prime} \log p_{j}^{\prime},
$$

(7)

where $p_{j}^{\prime}=\delta_{j}^{\prime} / \sum_{i=1}^{k} \delta_{j}^{\prime}$.

$p_{j}^{\prime}$ is the proportion of the i-th singular value in the entire singular value spectrum. Wavelet space feature entropy can distinguish the signal of the different time-frequency distribution quantitatively [8].

In the scale field $E$, wavelet transform, which is based on multiresolution analysis, can be used as a division of the signal energy. Signal $x(n)$ is decomposed with $J$ layer wavelet and reconstruction to get wavelet coefficients $D_{j}(k)$ and $A_{j}(k)$. The original signal sequence $\mathrm{x}$ can be expressed as the sum of the entire reconstruction coefficient.

$$
\begin{aligned}
& x(n)=D_{1}(n)+A_{1}(n)= \\
& D_{1}(n)+A_{1}(n)+A_{2}(n)= \\
& \sum_{j=1}^{J} D_{j}(n)+A_{J}(n)=\sum_{j=1}^{J+1} D_{j}(n)
\end{aligned}
$$

Under the scale $j$, the signal energy within a sliding window $C_{n}$ is expressed by

$$
E_{j C_{n}}=\sum_{k}\left|D_{j}(k)\right|^{2} \text {. }
$$

The total energy of the signal under $j+1$ layer scale within a sliding window $C_{n}$ is given by

$$
E_{C_{n}}=\sum_{j+1} E_{j C_{n}} .
$$

Wavelet energy spectrum entropy of original signal intercepted by sliding window is defined as

$$
S_{4}=\sum_{m=1}^{M} S_{m}
$$

where $S_{m}=-\sum_{i=1}^{J+1} p_{j} \log p_{j}$,

$$
p_{j}=\frac{E_{j C_{n}}}{E_{C_{n}}}
$$




\section{THE BASIC PRINCIPLE OF GREY CORRELATION DEGREE}

The gray relational analysis is a method that is used to make quantitative description and comparison of the development trend of the system changes. The purpose of gray relational analysis is to seek the key relationships between the various factors in the system and to identify the important factors affecting the target value [10]. The general steps of the analysis are as follows.

Identify the reference sequence

$$
X_{0}=\left\{X_{0}(k) \mid k=1,2, \cdots, m\right\} .
$$

Identify the comparative sequence,

$$
X_{i}=\left\{X_{i}(k) \mid k=1,2, \cdots, m\right\} \quad(i=1,2, \ldots, n) .
$$

Calculate the correlation coefficient after the mean treatment of the above sequence is done.

$$
\varepsilon_{i}(k)=\frac{\min _{i} \min _{k} \Delta_{i}(k)+\rho \max _{i} \max _{k} \Delta_{i}(k)}{\Delta_{i}(k)+\rho \max _{i} \max _{k} \Delta_{i}(k)},
$$

where $\Delta_{i}=\left|X_{i}-X_{0}\right|$.

$$
\rho-\text { distinguishing coefficient }, \quad \rho \in(0,1)
$$
usually $\rho=0.5$

$$
\min _{i} \min _{k} \Delta_{i}(k) \longrightarrow \text { the minimum rank difference }
$$$$
\max _{i} \max _{k} \Delta_{i}(k) \text { the maximum rank difference }
$$

Calculate the gray relational grade:

$$
r_{i}=1 / n \sum \varepsilon_{i}(k)
$$

The larger the value of gray relational degree, the closer the development trend of $X_{i}$ and $X_{0}$ the more similar they are.

\section{RESUlTS ANALYSIS}

The object of this experiment is the prototype of the inverter scroll compressor of scroll Machinery Research
Institute in Lanzhou University of Technology. The vibration testing points are as shown in Fig 1.



Figure 1. The selection of scroll compressor test points

In this study, the sampling frequency is $f_{1}=2000 \mathrm{~Hz}$, the motor speed is adjusted to $3120 \mathrm{r} / \mathrm{min}$ by the inverter. Number of Sampling is $N=1024$. When the scroll compressor is running smoothly, observe signals of 1-8 \# sensors. 1 \#, 5 \#, 7 \# and 8 \# vibration amplitude are relatively large. The scroll compressor vertex, scrolls meshing positions, electrical installation location and the bottom of the compressor have more severe vibration. For these test positions, the typical failures include rotor imbalance, failure of the scrolls, mechanical assembly loose and bearing loose.

After the sensor signal is analyzed, it is found that the entropy spectrum is sensitive to the detected vibration morphological changes and the vibrational energy distribution of the degree of uncertainty in the different scale space. Table I shows the 1 \#, 5 \#, 7 \#, 8 \# sensor three failure modes of the four entropy reference samples on the speed $3120 \mathrm{r} / \mathrm{min}$.

Fault sample is based on gray relational analysis method and the four failure modes of the information entropy of the different speed conditions. Test samples are taken from known 20 fault type data which have been stored in the

\begin{tabular}{|c|c|c|c|c|}
\hline & $\begin{array}{c}\text { Power spectral } \\
\text { entropy }\end{array}$ & $\begin{array}{c}\text { Singular spectrum } \\
\text { entropy }\end{array}$ & $\begin{array}{l}\text { Wavelet energy } \\
\text { spectrum entropy }\end{array}$ & $\begin{array}{c}\text { Spatial characteristics } \\
\text { of wavelet entropy }\end{array}$ \\
\hline Rotor unbalance $X_{1}$ & 1.6150 & 1.4980 & 1.0136 & 1.4892 \\
\hline $\begin{array}{l}\text { Scroll fault } X_{2} \\
\text { Mechanical assembly }\end{array}$ & 2.5837 & 1.9100 & 1.3084 & 2.5734 \\
\hline loose $X_{3}$ & 3.9224 & 2.2144 & 1.5797 & 2.7256 \\
\hline Bearing loose $X_{4}$ & 5.6675 & 2.3308 & 1.9445 & 2.9031 \\
\hline
\end{tabular}
vibration test platform to test the feasibility and accuracy of the method. Table II shows diagnostic results with the grey correlation degree analysis method for the four groups (five per group) of fault signal modes.

TABLE I. THE REFERENCE SEQUENCE VALUE OF FAULT SAMPLES OF THE INFORMATION ENTROPY 
TABLE II. THE COMPARISON OF VALUES OF GREY CORRELATION DEGREE BETWEEN THE TEST SIGNALS AND FAULT SAMPLES

\begin{tabular}{|c|c|c|c|c|c|c|c|c|c|}
\hline \multirow{2}{*}{$\begin{array}{c}\text { Test } \\
\text { Sample } \\
\text { Number }\end{array}$} & \multirow{2}{*}{$\begin{array}{c}\text { Different } \\
\text { Speeds } \\
\text { r/min }\end{array}$} & \multirow{2}{*}{$\begin{array}{c}\text { Power } \\
\text { Spectral } \\
\text { Entropy } \\
S_{1}\end{array}$} & \multirow{2}{*}{$\begin{array}{c}\text { Singular } \\
\text { Value } \\
\text { Entropy } \\
S_{2}\end{array}$} & \multirow{2}{*}{$\begin{array}{c}\text { Wavelet } \\
\text { Energy } \\
\text { Spectrum } \\
\text { Entropy } \\
S_{3}\end{array}$} & \multirow{2}{*}{$\begin{array}{c}\text { Spatial } \\
\text { Characteristic } \\
\text { Entropy of } \\
\text { Wavelet } \\
S_{4}\end{array}$} & \multicolumn{4}{|c|}{ Results of Gray Correlation Degree Analysis } \\
\hline & & & & & & $r_{1}$ & $r_{2}$ & $r_{3}$ & $r_{4}$ \\
\hline $\mathrm{X}_{1} \mathbf{1}$ & 2300 & 2.8765 & 1.4273 & 0.6281 & 2.1189 & 0.9078 & 0.4592 & 0.6724 & 0.7135 \\
\hline $\mathrm{X}_{1} 2$ & 2800 & 2.9122 & 1.9522 & 1.0129 & 2.5563 & 0.8965 & 0.5034 & 0.5675 & 0.6922 \\
\hline $\mathrm{X}_{1} 3$ & 3200 & 1.6213 & 1.5241 & 1.1215 & 1.4786 & 0.9263 & 0.5925 & 0.6782 & 0.7124 \\
\hline $\mathrm{X}_{1} 4$ & 3500 & 3.5721 & 2.8774 & 1.3129 & 3.1124 & 0.8629 & 0.6293 & 0.6231 & 0.3216 \\
\hline$X_{1} 5$ & 4000 & 1.9981 & 1.5327 & 0.9822 & 1.8325 & 0.9429 & 0.4276 & 0.3125 & 0.6298 \\
\hline $\mathrm{X}_{2} 1$ & 2300 & 3.6125 & 1.6127 & 0.8927 & 2.2343 & 0.3928 & 0.8110 & 0.5891 & 0.4873 \\
\hline$X_{2} 2$ & 2800 & 3.9573 & 2.6951 & 1.7324 & 3.3127 & 0.4562 & 0.8372 & 0.6173 & 0.5929 \\
\hline$X_{2} 3$ & 3200 & 2.5623 & 1.8983 & 1.6515 & 2.3038 & 0.7415 & 0.8642 & 0.7428 & 0.5853 \\
\hline$X_{2} 4$ & 3500 & 4.3740 & 3.2147 & 2.0170 & 4.1120 & 0.5520 & 0.9213 & 0.2980 & 0.6325 \\
\hline$X_{2} 5$ & 4000 & 3.1773 & 2.5320 & 1.7539 & 3.3118 & 0.6137 & 0.8726 & 0.7982 & 0.4728 \\
\hline$X_{3} 1$ & 2300 & 4.0127 & 1.9255 & 1.2488 & 3.5381 & 0.4587 & 0.5273 & 0.9163 & 0.3697 \\
\hline$X_{3} 2$ & 2800 & 4.2187 & 2.8922 & 2.2930 & 3.7328 & 0.7121 & 0.4273 & 0.8879 & 0.3127 \\
\hline$X_{3} 3$ & 3200 & 3.9013 & 2.1575 & 1.6213 & 2.7034 & 0.4234 & 0.5623 & 0.8135 & 0.4573 \\
\hline$X_{3} 4$ & 3500 & 4.4153 & 3.3123 & 2.5011 & 4.2785 & 0.4629 & 0.6724 & 0.8924 & 0.6241 \\
\hline$X_{3} 5$ & 4000 & 3.5527 & 2.7839 & 1.9787 & 3.4829 & 0.6128 & 0.5128 & 0.8763 & 0.4234 \\
\hline $\mathrm{X}_{4} 1$ & 2300 & 4.5273 & 2.3782 & 1.6785 & 3.9587 & 0.6922 & 0.4987 & 0.6129 & 0.8429 \\
\hline$X_{4} 2$ & 2800 & 4.6881 & 3.0174 & 2.7811 & 4.1314 & 0.5929 & 0.3895 & 0.3349 & 0.8959 \\
\hline $\mathrm{X}_{4} 3$ & 3200 & 5.8217 & 2.3302 & 1.9211 & 2.8978 & 0.4344 & 0.6129 & 0.6011 & 0.9427 \\
\hline$X_{4} 4$ & 3500 & 5.2725 & 3.9201 & 2.7963 & 4.9143 & 0.6321 & 0.5796 & 0.2238 & 0.9124 \\
\hline$X_{4} 5$ & 4000 & 4.0123 & 3.2010 & 2.1318 & 3.8781 & 0.3896 & 0.5552 & 0.4891 & 0.8538 \\
\hline
\end{tabular}

Where $r_{1}$ is the unbalance of the rotor, $r_{2}$ is the fault of the scroll, $r_{3}$ is the mechanical assembly loose, and $r_{4}$ is the bearing loose, they represent the gray correlation degree between the four kinds of typical fault sample and test patterns. The larger the value, the closer the test state is to the reference state. In four kinds of grey correlation degree, the largest value indicates that the test sample is belonging to the corresponding fault type. According to the data in Table 2, the diagnostic results are consistent with the actual fault type.

\section{CONCLUSION}

Where $r_{1}$ is the unbalance of the rotor, $r_{2}$ is the fault of the scroll, $r_{3}$ is the mechanical assembly loose, and $r_{4}$ is the bearing loose, they represent the gray correlation degree between the four kinds of typical fault sample and test patterns. The larger the value, the closer the test state is to the reference state. In four kinds of grey correlation degree, the largest value indicates that the test sample is belonging to the corresponding fault type. According to the data in Table 2, the diagnostic results are consistent with the actual fault type.

\section{ACKNOWLEDGMENT}

This study is supported by National Natural Science Foundation of China (Grant No. 50965011) and National Science Foundation of Gansu Province in China (Grant No. 1014RJZA025).

\section{REFERENCES}

[1] Zhenquan Liu. Scroll Type Fluid Machinery and Scroll Compressor [M]Beijing: China Machine Press,2009.

[2] Peng Bin, Liu Zhenquan, etc. Research on inverter scroll compressor test system [J].Control and Instruments in Chemical Industry, 2005, 32(3):58-61.

[3] Zhenquan Liu, Junshi Ren. Scroll compressor vibration timedomain and frequency-domain analysis [J].Fluid Machinery, 2000, 28(1):20-23.

[4] Liu Tao, Shao Hua. Vibrations signal analysis for inverter scroll compressor based on LabVIEW [J]. Electronic Measurement Technology, 2009, 32 (7):116-118.

[5] Shen Tao, Shuhong Huang, Shoumu Han, etc. Information entropy features of vibration signal of rotating machinery [J].Chinese Journal of Mechanical Engineering, 2001, 37(6):94-98.

[6] Xie Ping, Hongbin Lin, Wang Xiao, Liu Bin, etc. Study on a Multi-entropy Monitoring Method Used in Fault Diagnosis [J]. Chinese Journal of Scientific Instrument, 2004, 25(4):541-543.

[7] Chen Fei, Shuhong Huang, etc. Research on Diagnosis of Vibration Faults for Rotating Machinery Based on Distance of Information Entropy [J]. Journal of Vibration, Measurement \& Diagnosis, 2006,26(6):663-666.

[8] Junbao Geng, Shuhong Huang, Chen Fei. Rotating machinery fault diagnosis based on close degree to information entropy[J].Journal of Huazhong University of Science and Technology(Natural Science Edition),2006,34(11):93-95.

[9] Jingchuan Zhang, Zhoumo Zeng, Feng Ping, etc. A recognition method with wavelet energy spectrum and wavelet information entropy for abnormal vibration events of a petroleum pipeline [J]. Journal of Vibration and Shock, 2010, 29 (5):1-4.

[10] Julong Deng. The basis of gray theory [M] . Wuhan: Huazhong University of Science and Technology Press, 2002. 